\title{
Safety of Inpatient Dofetilide Initiation per Cardiology Services: A Retrospective Review
}

\author{
Carrie Cicirale ${ }^{1}$ \\ ${ }^{1}$ Mercy Medical Center Homecare and Hospice LLC
}

May 21, 2020

\begin{abstract}
Background Dofetilide is an antiarrhythmic medication that has the potential to cause life threatening arrhythmias, such as Torsade de pointes. The 2014 ACC/AHA/HRS guidelines for the management of patients with atrial fibrillation state that patients need to meet certain criteria to be initiated on dofetilide. Patients who are not initiated on this therapy according to the guideline criteria are likely to be at higher risk of adverse reactions. Methods This is a single center, retrospective chart review of patients who were initiated on dofetilide from July 2016-December 2019. Patients included in the study were initiated on dofetilide as a new antiarrhythmic and monitored inpatient for three days. The primary outcome was a composite of incidence of cardiac arrhythmias, cardiac arrest, cardiac death, and cardiac related hospital readmission. Results There were 224 patients included in the analysis: 190 patients who were initiated on dofetilide inappropriately and 34 that were initiated appropriately. The primary outcome (composite of cardiac arrhythmia, cardiac arrest, cardiac death, and hospital readmission) was statistically significant with more patients experiencing an outcome in the group initiated inappropriately. Discussion Patients are placed at a higher risk of adverse reactions when this potentially dangerous antiarrhythmic medication is not used according to the protocol set forth by the guidelines. Conclusion Practitioners should use caution when prescribing dofetilide. Other antiarrhythmic medications or non-pharmacologic options should be considered due to the incidence of these dangerous adverse reactions.
\end{abstract}

\section{Hosted file}

Manuscript Carrie Cicirale.pdf available at https://authorea.com/users/324940/

articles/453009-safety-of-inpatient-dofetilide-initiation-per-cardiology-services-a-

retrospective-review 


\begin{tabular}{|c|c|c|c|}
\hline \multicolumn{4}{|c|}{ Table 1: Baseline Characteristics } \\
\hline & Not Appropriate & Appropriate & \\
\hline Patient Characteristic & $(n=190)$ & $(n=34)$ & P-value \\
\hline Age (Years) & & & 0.311 \\
\hline Mean (SD) & $71.3(10.32)$ & $69.4(9.27)$ & \\
\hline Gender - n (\%) & & & 0.470 \\
\hline Female & $91(47.9)$ & $14(41.2)$ & \\
\hline Male & $99(52.1)$ & $20(58.8)$ & \\
\hline BMI (kg/m2) & & & 0.904 \\
\hline Mean (SD) & $32.5(9.13)$ & $32.3(7.50)$ & \\
\hline Diagnosis - n (\%) & & & 0.619 \\
\hline Atrial fibrillation & $126(66.3)$ & $25(73.5)$ & \\
\hline Atrial fibrillation/Atrial flutter & $63(33.2)$ & $9(26.5)$ & \\
\hline Atrial flutter & $1(0.5)$ & 0 & \\
\hline \multicolumn{4}{|l|}{ Cardiac History - n (\%) } \\
\hline Hypertension & $168(88.4)$ & $33(97.1)$ & 0.215 \\
\hline Hyperlipidemia & $141(74.2)$ & $23(67.6)$ & 0.426 \\
\hline Heart Failure & $116(61.1)$ & $23(67.6)$ & 0.466 \\
\hline Coronary Artery Disease & $86(45.3)$ & $14(41.2)$ & 0.659 \\
\hline $\mathrm{CrCl}$ (Baseline) & & & 0.943 \\
\hline Mean (SD) & $65.9(26.80)$ & $66.3(28.46)$ & \\
\hline
\end{tabular}

